Annals of Pure and Applied Mathematics

Vol. 16, No. 2, 2018, 295-304

ISSN: 2279-087X (P), 2279-0888(online)

Published on 17 February 2018

www.researchmathsci.org

DOI: http://dx.doi.org/10.22457/apam.v16n2a5

Annals of

Pure and Applied

Mathematics

\title{
A Note on Fuzzy Bi-Ideals in Ternary Semigroups
}

\author{
U.Nagi Reddy ${ }^{1}$, K.Rajani and ${ }^{2}$ and G. Shobhalatha ${ }^{3}$
}

${ }^{1}$ Department of Mathematics, Rayalaseema University, Kurnool - 518002

AP, India

${ }^{2,3}$ Department of Mathematics, Sri Krishnadevaraya University

Ananthapuramu - 515003, AP, India

email: nagireddyppr@gmail.com

Received 2 January 2018; accepted 26 January 2018

Abstract. The prime fuzzy bi-ideals of semigroups are introduced by Shabir, Jun and Bano. They characterized those semigroups for which each fuzzy bi-ideal is semiprime and also characterized those semigroups for which each fuzzy bi-ideal is strongly prime. In this paper, we prove some properties of fuzzy bi-ideals in ternary semigroups and the relation between fuzzy quasi ideals and fuzzy bi-ideals is considered.

Keywords: Ternary semigroup, fuzzy set, fuzzy ternary semigroup, fuzzy ideal, fuzzy quasi ideal, fuzzy bi-ideal, characteristic function.

\section{AMS Mathematics Subject Classification (2010): 47D03}

\section{Introduction}

Lehmer introduced the ternary algebraic system in 1932, and after such structures were studied by Kasner. In 1965, ideal theory in ternary semigroups studied by Sioson [16]. After the introduction of fuzzy sets by Zadeh [9] reconsideration of the concept of classical mathematics began. Fuzzy set has an important impact over the field of mathematical research in both theory and application. It has found manifold applications in mathematics and related areas. Kuroki introduced and studied the notion of fuzzy semigroups. He also studied the concept of fuzzy bi-ideals [6] (1979) and fuzzy quasiideals (1982) of semigroups. Since then many papers have been published in the field of fuzzy algebra $[1-5,7,8,10-15,17,18]$. Many researchers conducted the researches on the generalizations of the notions of fuzzy sets with huge applications in computer, logics and many branches of pure and applied mathematics.

\section{Basic definitions and preliminaries}

Definition 2.1. A non-empty set $T$ is said to be ternary semigroup if there exists a ternary operation : $: T \times T \times T \rightarrow T$ written as $(a, b, c) \rightarrow a . b . c$ satisfies the following identity $(a b c) d e=a(b c d) e=a b(c d e)$ for all $a, b, c, d, e \in T$. 
U. Nagi Reddy, K Rajani and G. Shobhalatha

Example 1. Let $T=\left\{\left[\begin{array}{ll}0 & 0 \\ 0 & 0\end{array}\right],\left[\begin{array}{ll}1 & 0 \\ 0 & 1\end{array}\right],\left[\begin{array}{ll}1 & 0 \\ 0 & 0\end{array}\right],\left[\begin{array}{ll}0 & 1 \\ 0 & 0\end{array}\right],\left[\begin{array}{ll}0 & 0 \\ 1 & 0\end{array}\right],\left[\begin{array}{ll}0 & 0 \\ 0 & 1\end{array}\right]\right\}$ then $T$ is a ternary semigroup under usual multiplication.

Definition 2.2. A non-empty subset $A$ of a ternary semigroup $T$ is called a ternary subsemigroup of $T$ if $A A A \subseteq A$.

Definition 2.3. A non-empty subset $A$ of a ternary semigroup $T$ is called a quasi ideal in $T$ if

$$
(A T T) \cap(T A T) \cap(T T A) \subseteq A \text { and }(A T T) \cap(T T A T T) \cap(T T A) \subseteq A .
$$

Definition 2.4. A ternary sub semigroup $A$ of a ternary semigroup $T$ is said to be a biideal in $T$ if $A T A T A \subseteq A$.

Definition 2.5. Let $T$ be a non-empty set. A fuzzy subset of a ternary semigroup $T$ is a function $\mu: T \rightarrow[0,1]$.

Definition 2.6. Let $\mu$ be a fuzzy subset of a non-empty set $T$ for any $t \in[0,1]$, the subset $\mu_{t}=\{x \in T: \mu(x) \geq t\}$ of $T$ is called a level set of $\mu$.

Definition 2.7. For any two fuzzy subsets $\mu_{1}$ and $\mu_{2}$ of a non-empty set $T$, the union and the intersection of $\mu_{1}$ and $\mu_{2}$ denoted by $\mu_{1} \cup \mu_{2}$ and $\mu_{1} \cap \mu_{2}$ are fuzzy subsets of $T$ and defined as

$\left(\mu_{1} \cup \mu_{2}\right)(x)=\max \left\{\mu_{1}(x), \mu_{2}(x)\right\}=\mu_{1}(x) \vee \mu_{2}(x)$ and $\left(\mu_{1} \cap \mu_{2}\right)(x)=\min \left\{\mu_{1}(x), \mu_{2}(x)\right\}=\mu_{1}(x) \wedge \mu_{2}(x) \quad$ for all $x \in T$.

Where $\vee$ denotes maximum or supremum and $\wedge$ denotes minimum or infimum.

Definition 2.8. Let $\mu_{1}, \mu_{2}$ and $\mu_{3}$ are any three fuzzy sets of a ternary semigroup $T$. Then their fuzzy product $\mu_{1} \circ \mu_{2} \circ \mu_{3}$ is defined by

$$
\left(\mu_{1} \circ \mu_{2} \circ \mu_{3}\right)(a)= \begin{cases}\mathrm{V}_{a=x y z}\left\{\mu_{1}(x) \wedge \mu_{2}(y) \wedge \mu_{3}(z)\right\} & \text { if a is expressible as } a=x y z \\ 0 & \text { otherwise }\end{cases}
$$

Definition 2.9. A fuzzy set $\mu$ of a ternary semigroup $T$ is called a fuzzy ternary subsemigroup of $T$ if $\mu(x y z) \geq\{\mu(x) \wedge \mu(y) \wedge \mu(z)\}$ for all $x, y, z \in T$.

Definition 2.10. A fuzzy ternary sub semigroup $\mu$ of a ternary semigroup $T$ is called a fuzzy bi-ideal in $T$ if $\mu(x m y n z) \geq\{\mu(x) \wedge \mu(y) \wedge \mu(z)\}$ for all $x, y, z m, n \in T$. 
A Note on Fuzzy Bi-Ideals in Ternary Semigroups

Definition 2.11. A fuzzy set $\mu$ of a ternary semigroup $T$ is called a fuzzy left (right, lateral) ideal in $T$ if $\mu(x y z) \geq \mu(z),(\mu(x y z) \geq \mu(x), \mu(x y z) \geq \mu(y))$ for all $x, y, z \in T$..

Definition 2.12. A fuzzy set $\mu$ of a ternary semigroup $T$ is a fuzzy ideal in $T$ if it is fuzzy left, right and lateral ideal in $T$.

Definition 2.13. Let $A$ be a non-subset of a ternary semigroup $T$. Then the

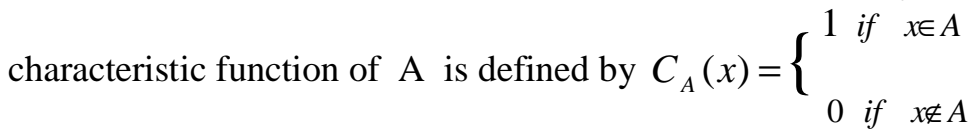

We denote the characteristic function $C_{T}$ of $T$. i.e., $T=C_{T}$ thus $T(x)=1$ for all $x \in T$.

Definition 2.14. A fuzzy set $\mu$ of a ternary semigroup $T$ is called a fuzzy quasi ideal of $T$ if $(T \circ T \circ \mu) \cap(T \circ \mu \circ T) \cap(\mu \circ T \circ T) \subseteq \mu$ and

$(T \circ T \circ \mu) \cap(T \circ T \circ \mu \circ T \circ T) \cap(\mu \circ T \circ T) \subseteq \mu$.

i.e., $[(T \circ T \circ \mu) \cap(T \circ \mu \circ T) \cap(\mu \circ T \circ T)](a) \leq \mu(a)$ and $[(T \circ T \circ \mu) \cap(T \circ T \circ \mu \circ T \circ T) \cap(\mu \circ T \circ T)](a) \leq \mu(a)$

\section{Main results}

Theorem 3.1. A non-empty subset $A$ of a ternary semigroup $T$ is a bi- ideal in $T$ if and only if characteristic function $C_{A}$ of $A$ is a fuzzy bi - ideal in $T$.

Proof: Let $C_{A}$ is a characteristic function of $A$ in ternary semigroup $T$.

Assume that $A$ is a bi-ideal in $T$.Then we have $A T A T A \subseteq A$.

Now consider $C_{A} \circ T \circ C_{A} \circ T \circ C_{A}=C_{A} \circ C_{T} \circ C_{A} \circ C_{T} \circ C_{A}$

$$
=C_{\text {ATATA }} \subseteq C_{A}
$$

$$
C_{A} \circ T \circ C_{A} \circ T \circ C_{A} \subseteq C_{A}
$$

Therefore $C_{A}$ is a fuzzy bi ideal in $T$.

Conversely, suppose $C_{A}$ is fuzzy bi-ideal in $T$.

Then $C_{A} \circ T \circ C_{A} \circ T \circ C_{A} \subseteq C_{A}$

Let $x \in$ ATATA. Then $C_{A}(x) \geq\left(C_{A} \circ T \circ C_{A} \circ T \circ C_{A}\right)(x)$

$$
=\left(C_{A} \circ C_{T} \circ C_{A} \circ C_{T} \circ C_{A}\right)(x)
$$$$
=C_{\text {ATATA }}(x)
$$$$
\geq 1(\because x \in(\text { ATATA })
$$

$$
C_{A}(x) \geq 1 \Rightarrow x \in A
$$

Therefore $A T A T A \subseteq A$.

Hence $A$ is a bi-ideal in a ternary semi group $T$. 
U. Nagi Reddy, K Rajani and G. Shobhalatha

Theorem 3.2. If $\mu$ be a fuzzy bi-ideal in a ternary semigroup $T$, then the level set $\mu_{t}$ is a bi -ideal in $T$ for every $t \in[0,1]$.

Proof: Let $\mu$ be a fuzzy subset of a ternary semigroup $T$ and let $x \in T$. For $t \in[0,1]$, let $u \in \mu_{t} T \mu_{t} T \mu_{t}$, where $\mu_{t}$ be the level set of $\mu$. Then there exists $x, y, z \in \mu_{t}$, $m, n \in T$ and such that $u=x m y n z$.

Consider $[\mu \circ T \circ(\mu \circ T \circ \mu)](u)=\underset{u=a b c}{\vee}\{\mu(a) \wedge \mu(b) \wedge \mu(c)\}$

$$
\begin{aligned}
& =\underset{u=a b c}{\vee}\{\mu(a) \wedge T(b) \wedge\{\underset{c=d e f}{\vee}\{\mu(d) \wedge T(e) \wedge \mu(f)\}\}\} \\
& =\bigvee_{u=a b d e f}\{\mu(a) \wedge 1 \wedge \mu(d) \wedge 1 \wedge \mu(f)\} \\
& =\underset{x m y n z=a b d e f}{\bigvee}\{\mu(a) \wedge \mu(d) \wedge \mu(f)\} \\
& =\mu(x) \wedge \mu(y) \wedge \mu(z) \\
& \geq t \wedge t \wedge t=t \\
& {[\mu \circ T \circ(\mu \circ T \circ \mu)](u) \geq t}
\end{aligned}
$$

Since $\mu$ is a fuzzy bi-ideal in $T \Rightarrow \mu(u) \geq t$ for all $u \in T$

$\Rightarrow u \in \mu_{t} \Rightarrow \mu_{t} T \mu_{t} T \mu_{t} \subseteq \mu_{t}$.

Hence $\mu_{t}$ is a bi- ideal in $T$.

Theorem 3.3. Every fuzzy quasi ideal in a ternary semigroup $T$ is a fuzzy bi -ideal in $T$.

Proof: Suppose $\mu$ is fuzzy quasi ideal in a ternary semigroup $T$. Then we have $(T \circ T \circ \mu) \cap(T \circ \mu \circ T) \cap(\mu \circ T \circ T) \subseteq \mu$ and

$(T \circ T \circ \mu) \cap(T \circ T \circ \mu \circ T \circ T) \cap(\mu \circ T \circ T) \subseteq \mu$.

Consider $\mu(x y z) \geq[(T \circ T \circ \mu) \cap(T \circ \mu \circ T) \cap(\mu \circ T \circ T)](x y z)$

$\geq(T \circ T \circ \mu)(x y z) \cap(T \circ \mu \circ T)(x y z) \cap(\mu \circ T \circ T)(x y z)$

$\geq\left[\bigvee_{x z=a b c}\{T(a) \wedge T(b) \wedge \mu(c)\}\right] \wedge\left[\bigvee_{x y z=u v w}\{T(u) \wedge T(v) \wedge \mu(w)\}\right]$

$$
\wedge\left[\bigvee_{x y z=r s t}\{\mu(r) \wedge T(s) \wedge T(t)\}\right]
$$

$\geq[T(x) \wedge T(y) \wedge \mu(z)] \wedge[T(x) \wedge \mu(y) \wedge T(z)] \wedge[\mu(x) \wedge T(y) \wedge T(z)]$

$\geq[1 \wedge 1 \wedge \mu(z)] \wedge[1 \wedge \mu(y) \wedge 1] \wedge[\mu(x) \wedge 1 \wedge 1]$

$\geq \mu(z) \wedge \mu(y) \wedge \mu(x)$

$\geq \mu(x) \wedge \mu(y) \wedge \mu(z)$

$\mu(x y z) \geq[\mu(x) \wedge \mu(y) \wedge \mu(z)]$

Therefore $\mu$ is a fuzzy ternary sub semigroup of $T$.

Again consider

$\mu(x m y n z) \geq[(T \circ T \circ \mu) \cap(T \circ T \circ \mu \circ T \circ T) \cap(\mu \circ T \circ T)](x m y n z)$ 
A Note on Fuzzy Bi-Ideals in Ternary Semigroups

$$
\begin{aligned}
& \geq[(T \circ T \circ \mu)(x m y n z) \cap(T \circ T \circ \mu \circ T \circ T)(\text { xmynz }) \cap(\mu \circ T \circ T)(\text { xmynz })] \\
& \geq[\underset{\text { xmynz=abcde }}{\vee}\{T(a) \wedge T(b d c) \wedge \mu(e)\}] \wedge \\
& \qquad \underset{(x m y n z)=p q r s t}{\vee}\{T(p) \wedge T(q) \wedge \mu(r) \wedge T(r) \wedge T(t)\}] \\
& \qquad[\underbrace{}_{x m y n z=i j k l o}\{\mu(i) \wedge T(j k l) \wedge T(o)\}] \\
& \geq[T(x) \wedge T(m y n) \wedge \mu(z)] \wedge[T(x) \wedge T(m) \wedge \mu(y) \wedge T(n) \wedge T(z)] \\
& \geq[1 \wedge 1 \wedge \mu(z)] \wedge[1 \wedge 1 \wedge \mu(y) \wedge 1 \wedge 1] \wedge[\mu(x) \wedge 1 \wedge 1] \\
& \geq \mu(z) \wedge \mu(y) \wedge \mu(x) \\
& \mu(x m y n z) \geq \mu(x) \wedge \mu(y) \wedge \mu(z)
\end{aligned}
$$

Therefore $\mu$ is a fuzzy bi - ideal in $T$.

Theorem 3.4. Let $\mu$ be a fuzzy bi-ideal in a ternary semigroup $T$. Then the fuzzy subset $\mu^{*}$ defined by $\mu^{*}=\mu(x)+1-\mu(0)$ for all $x \in T$ is also a fuzzy bi- ideal of $T$. Proof: Given that $T$ be a ternary semigroup and $\mu$ be a fuzzy bi-ideal in $T$. Then $\mu$ is a fuzzy ternary sub semigroup of $T$. That is for all $x, y, z \in T$,

$$
\begin{aligned}
& \mu(x y z) \geq \mu(x) \wedge \mu(y) \wedge \mu(z) \\
& \quad \text { or } \mu(x y z) \geq \min \{\mu(x), \mu(y), \mu(z)\} .
\end{aligned}
$$

and $\mu(a m y n z) \geq \min \{\mu(a), \mu(b), \mu(c)\} \quad$ for all $a, b, c, m, n \in T$.

Let $\mu^{*}$ be a fuzzy subset of $\mathrm{T}$ where $\mu^{*}=\mu(x)+1-\mu(0)$ for all $x \in T$.

we have to prove that $\mu^{*}$ is a fuzzy bi - ideal of $T$.

(i) Let $x, y, z \in T$

We have $\mu^{*}(x y z)=\mu(x y z)+1-\mu(0)$

$$
\begin{aligned}
& \geq \min \{\mu(x), \mu(y), \mu(z)\}+1-\mu(0) \\
& =\min \{\mu(x)+1-\mu(0), \mu(y)+1-\mu(0), \mu(z)+1-\mu(0)\} \\
& =\min \left\{\mu^{*}(x), \mu^{*}(y), \mu^{*}(z)\right\} \\
\mu^{*}(x y z) & \geq \min \left\{\mu^{*}(x), \mu^{*}(y), \mu^{*}(z)\right\}
\end{aligned}
$$

Therefore $\mu^{*}$ is a fuzzy ternary sub semigroup of $T$.

(ii) Let $a, b, c, m, n \in T$

We have $\mu^{*}(a m b n c)=\mu(a m b n c)+1-\mu(0)$

$$
\begin{aligned}
& \geq \min \{\mu(a), \mu(b), \mu(c)\}+1-\mu(0) \\
= & \min \{\mu(a)+1-\mu(0), \mu(b)+1-\mu(0), \mu(c)+1-\mu(0)\} \\
= & \min \left\{\mu^{*}(a), \mu^{*}(b), \mu^{*}(c)\right\}
\end{aligned}
$$


U. Nagi Reddy, K Rajani and G. Shobhalatha

$$
\mu^{*}(\text { ambnc }) \geq \min \left\{\mu^{*}(a), \mu^{*}(b), \mu^{*}(c)\right\}
$$

Therefore $\mu^{*}$ is a fuzzy bi -ideal in $T$.

Theorem 3.5. Let $T$ be a left zero ternary semigroup and $\mu$ be a fuzzy left ideal in $T$. Then $\mu(x)=\mu(z)$ for all $x, y, z \in T$.

Proof: Let $T$ be a left zero ternary semigroup.

i.e., $x, y, z \in T \Rightarrow x y z=x$ and $z y x=z$.

since $\mu$ is fuzzy left ideal in $T$.

Consider $\quad \mu(x)=\mu(x y z) \geq \mu(z)$

$$
\mu(x) \geq \mu(z)
$$

and we have $\mu(x)=\mu(z y x) \geq \mu(x)$

$$
\mu(z) \geq \mu(x)
$$

from (1) and (2), We have $\mu(x)=\mu(z)$ for all $x, y, z \in T$.

Theorem 3.6. A non-empty fuzzy subset $\mu$ of a ternary semigroup $T$ is a fuzzy ternary sub semigroup of $T$ if and only if $\mu \circ \mu \circ \mu \subseteq \mu$.

Proof: Let $\mu$ be a non- empty fuzzy subset of a ternary semigroup $T$.

Suppose $\mu \circ \mu \circ \mu \subseteq \mu$

we have to prove that $\mu$ is a fuzzy ternary sub semigroup of $T$.

$$
\mu(x y z) \geq \mu(x) \wedge \mu(y) \wedge \mu(z) \text { for all } x, y, z \in T .
$$

Let $x, y, z \in T$, such that $p=x y z$

Consider $\mu(x y z) \geq(\mu \circ \mu \circ \mu)(x y z)$

$$
\begin{gathered}
\quad \underset{p=a b c}{\vee}\{\mu(a) \wedge \mu(b) \wedge \mu(c)\} \\
=\underset{x y z=a b c}{\vee}\{\mu(a) \wedge \mu(b) \wedge \mu(c)\} \\
=\mu(x) \wedge \mu(y) \wedge \mu(z) \\
\mu(x y z) \geq\{\mu(x) \wedge \mu(y) \wedge \mu(z)\} .
\end{gathered}
$$

Conversely, assume that $\mu$ is a fuzzy ternary sub semigroup of $T$.

$$
\text { i.e., } \mu(x y z) \geq\{\mu(x) \wedge \mu(y) \wedge \mu(z)\} \text {. }
$$

We have to prove that $\mu \circ \mu \circ \mu \subseteq \mu$.

$$
\text { i.e., }(\mu \circ \mu \circ \mu)(a) \leq \mu(a) \quad \text { for all } a \in T
$$

Let $a=x y z$ for all $x, y, z \in T$.

$$
\text { Consider } \begin{aligned}
(\mu \circ \mu \circ \mu)(a) & =\underset{a=p q r}{\vee}\{\mu(p) \wedge \mu(q) \wedge \mu(r)\} \\
& =\underset{x y z=p q r}{\vee}\{\mu(p) \wedge \mu(q) \wedge \mu(r)\} \\
& =\{\mu(x) \wedge \mu(y) \wedge \mu(z)\} \\
& \leq \mu(x y z)
\end{aligned}
$$


A Note on Fuzzy Bi-Ideals in Ternary Semigroups

$=\mu(a)$

$(\mu \circ \mu \circ \mu)(a) \leq \mu(a) \quad \Rightarrow \quad \mu \circ \mu \circ \mu \subseteq \mu$.

If $a \neq x y z$ then $(\mu \circ \mu \circ \mu)(a)=o \leq \mu(a) \quad \Rightarrow \quad(\mu \circ \mu \circ \mu)(a) \leq \mu(a)$.

Therefore $\mu \circ \mu \circ \mu \subseteq \mu$.

Theorem 3.7. For any nonempty fuzzy subset $\mu$ of a ternary semigroup $T$, the following conditions are equivalent.

(i) $\mu$ is a fuzzy bi-ideal in $T$.

(ii) $\mu \circ \mu \circ \mu \subseteq \mu$ and $\mu \circ C_{T} \circ \mu \circ C_{T} \circ \mu \subseteq \mu$ where $C_{T}$ is the characteristic function of $T$.

Proof: Let $T$ be a ternary semigroup and let $\mu$ be a fuzzy subset of $T$.

First to prove that (i) $\Rightarrow$ (ii):

Let $\mu$ is a fuzzy bi-ideal in $T$ then $\mu$ is a fuzzy ternary sub semigroup of $T$. i.e., $\mu(x y z) \geq\{\mu(x) \wedge \mu(y) \wedge \mu(z)\} \quad$ for all $x, y, z \in T$.

and $\mu($ amynz $) \geq\{\mu(a) \wedge \mu(b) \wedge \mu(c)\} \quad$ for all $a, b, c, m, n \in T$..

From the theorem 3.6, we have $\mu \circ \mu \circ \mu \subseteq \mu$.

To prove only $\mu \circ C_{T} \circ \mu \circ C_{T} \circ \mu \subseteq \mu$

i.e., $\left(\mu \circ C_{T} \circ \mu \circ C_{T} \circ \mu\right)(a) \leq \mu(a)$ for all $a \in T$.

Let $a=x m y n z$ for all $x, y, z, m, n, p, q, r \in T$.

Consider

$$
\begin{aligned}
&\left.\mu \circ C_{T} \circ \mu \circ C_{T} \circ \mu\right)\left.(a)=\underset{\text { xmynz }=\text { puqvr }}{\vee}\left\{\mu(p) \wedge C_{T}(u) \wedge \mu(q) \wedge C_{T}(v) \wedge \mu\right)(r)\right\} \\
&=\left\{\mu(x) \wedge C_{T}(m) \wedge \mu(y) \wedge C_{T}(n) \wedge \mu(z)\right\} \\
&=\{\mu(x) \wedge 1 \wedge \mu(y) \wedge 1 \wedge \mu(z)\} \\
&=\{\mu(x) \wedge \mu(y) \wedge \mu(z)\} \\
& \leq \mu(\text { xmynz }) \\
&= \mu(a) \\
& \Rightarrow\left(\mu \circ C_{T} \circ \mu \circ C_{T} \circ \mu\right)(a) \leq \mu(a) .
\end{aligned}
$$

Therefore $\mu \circ C_{T} \circ \mu \circ C_{T} \circ \mu \subseteq \mu$

Next to prove that (ii) $\Rightarrow$ (i):

We assume that $\mu \circ \mu \circ \mu \subseteq \mu \rightarrow$ (1) and $\mu \circ C_{T} \circ \mu \circ C_{T} \circ \mu \subseteq \mu \rightarrow$ (2).

From theorem 3.6 and by equation (1), we have $\mu$ is a fuzzy ternary sub semigroup of $T$.

It is enough to prove that $\mu($ a mynz $) \geq \min \{\mu(a), \mu(b), \mu(c)\}$. 
U. Nagi Reddy, K Rajani and G. Shobhalatha

Consider $\mu(x$ mynz $) \geq\left\{\mu \circ C_{T} \circ \mu \circ C_{T} \circ \mu\right\}$ (xmyn z).

$$
\begin{aligned}
& =\underset{\text { xmynz }=\text { puqvr }}{\vee}\left\{\mu(p) \wedge C_{T}(u) \wedge \mu(q) \wedge C_{T}(v) \wedge \mu(r)\right\} . \\
& =\left\{\mu(x) \wedge C_{T}(m) \wedge \mu(y) \wedge C_{T}(n) \wedge \mu(z)\right\} . \\
& =\{\mu(x) \wedge 1 \wedge \mu(y) \wedge 1 \wedge \mu(z)\} . \\
& =\{\mu(x) \wedge \mu(y) \wedge \mu(z)\} .
\end{aligned}
$$

$\mu(x m y n z) \geq\{\mu(x) \wedge \mu(y) \wedge \mu(z)\}$ for all $a, b, c, m, n \in T$.

Therefore $\mu$ is a fuzzy bi - ideal in $T$.

Definition 3.8. Let $T_{1}$ and $T_{2}$ be two ternary semigroups. A mapping

$f:\left(T_{1}, *_{1}\right) \rightarrow\left(T_{2}, *_{2}\right)$ is called a ternary homomorphism if $f\left(x *_{1} y *_{1} z\right)=\left(f(x) *_{2} f(y) *_{2} f(z)\right) \quad$ for all $x, y, z \in T$.

Definition 3.9. Let $f$ be a mapping from a set $\mathrm{X}$ to $\mathrm{Y}$ and $\mu$ be a fuzzy subset of $\mathrm{Y}$, then the pre image of $\mu$ under $f$, denoted by $f^{-1}(\mu)$, is defined as

$$
f^{-1}(\mu)(x)=\mu(f(x)) \text { for all } x \in X .
$$

Theorem 3.10. Let $f: T_{1} \rightarrow T_{2}$ be a homomorphism of ternary semigroups. If $\mu$ is a fuzzy bi- ideal in $T_{2}$ then the pre image $f^{-1}(\mu)$ is a fuzzy bi-ideal in $T_{1}$.

Proof: Let $T_{1}$ and $T_{2}$ be two ternary semigroups and given that $f: T_{1} \rightarrow T_{2}$ is a homomorphism.

Then $f(x y z)=(f(x) f(y) f(z)) \quad$ for all $\quad x, y, z \in T_{1}$.

Let $\mu$ be a fuzzy bi - ideal in $T_{2}$.

We have to prove that $f^{-1}(\mu)$ is a fuzzy bi-ideal in $T_{1}$.

Consider $f^{-1}(\mu(x y z))=\mu(f(x y z))$.

$$
\begin{aligned}
& =\mu(f(x) f(y) f(z)) . \\
& \geq \min \{\mu(f(x)), \mu(f(y)) f(\mu(z))\} . \\
& =\min \left\{f^{-1}(\mu(x)), f^{-1}(\mu(y)) f^{-1}(\mu(z))\right\} . \\
f^{-1}(\mu(x y z)) & \geq \min \left\{f^{-1}(\mu(x)), f^{-1}(\mu(y)) f^{-1}(\mu(z))\right\} .
\end{aligned}
$$

Therefore, $f^{-1}(\mu)$ is a fuzzy ternary sub semigroup of $T$.

Let $x, y, z, m, n \in T_{1}$

Again consider $f^{-1}(\mu(x m y n z))=\mu(f(x m y n z))$

$$
=\mu(f(x) f(m) f(y) f(n) f(z)) .
$$


A Note on Fuzzy Bi-Ideals in Ternary Semigroups

$$
\begin{gathered}
\geq \mu(f(x)) \mu(f(y)) \mu(f(z))) . \\
=\min \left\{f^{-1}(\mu(x)) f^{-1}(\mu(y)) f^{-1}(\mu(z))\right\} . \\
f^{-1}(\mu(x m y m z)) \geq \min \left\{f^{-1}(\mu(x)), f^{-1}(\mu(y)), f^{-1}(\mu(z))\right\} .
\end{gathered}
$$

Therefore $f^{-1}(\mu)$ is a fuzzy bi - ideal in ternary semigroup $T_{1}$.

\section{Conclusions}

We introduced the notion of fuzzy ideal, fuzzy quasi ideal, fuzzy bi-ideal in an ternary semigroup and studied their properties and relations between them. We characterize the fuzzy bi-ideals in an ternary semigroup with respect to bi ideals. In continuous of this paper we propose to study fuzzy bi-ideals over Ternary semigroups.

Acknowledgement. We are thankful to the reviewer for the reviewing our paper. Your suggestions and Comment are very helpful in making further improvement if any. Thank you for your support.

\section{REFERENCES}

1. M.Amyari and M.S.Moslehain, Approximate Homomorphisms of ternary semigroups, arXiv: maths-ph/0511039v1 10 Nov 2005.

2. V.N.Dixit and S.Dewan, A note on quasi ideals and bi- ideals in ternary semigroups, Internet, J.Math and Math. Sci., 18(3) (1995) 501-508.

3. T.K.Dutta, S.Kar and B.K.Maity, On ideals in regular ternary semigroup, Discuss. Math. Gen. Algebra Appl., 28 (2008) 147-159.

4. Hai-zhen Liu and P.Yang, Rees nilpotent radical and semisimple semigroups, Annals of Pure and Applied Mathematics, 9(1) (2015) 53-65.

5. S.Kar and P.Sarkar, Fuzzy quasi ideals and fuzzy bi- ideals of ternary semigroups, Annals of Fuzzy Mathematics and Informatics, 4(2) (2012) 407-423.

6. N.Kuroki, Fuzzy bi-ideals in Semigroups, Comment. Math. Univ. St. Paul., 28 (1979) 17-21.

7. D.H.Lehmer, A ternary analogue of abelian groups, Amer. J. Math., 54 (1932) 329-338.

8. L.A.Zadeh, Fuzzy sets, Inform. Control., 8 (1965) 338-353.

9. U.Nagi Reddy and G. Shobhalatha, Some characterizations of ternary semigroups, International Journal of Scientific \& Engineering Research, 6(9) (2015) ????

10. U.Nagi Reddy and G. Shobhalatha, Note on fuzzy weakly completely prime - ideals in ternary semigroups, International Journal of Mathematical Archive, 7(5) (2016) 193-198.

11. U.Nagi Reddy, K.V.Subrahmanyam and G.Shobhalatha, A note on $£$ - classes on right regular partiallyordered ternary semigroups, Open Journal of Applied \& Theoretical Mathematics, 2(4) (2016) 535 547.

12. N.Kehayopulu and M.Tsingelis, Fuzzy ideals in ordered semigroups, Quasi Groups and Related System, 15 (2007) 279-289.

13. Parinyawat Choosuwan and Rannason Chinram, A study on quasi ideals in ternary semigroups, International Journal of pure and Applied Mathematics, 77(5) (2012) 639-647. 
U. Nagi Reddy, K Rajani and G. Shobhalatha

14. D.R.Prince Wiliams, K.B.Latha and E.Chandrasekarn, Fuzzy bi-ideal in $\Gamma-$ semigroups, Hacettepe Journal of Mathematics and Statistics, 38 (1) (2009) 1-15.

15. M.Shabir, Y.B.Jun and M.Bano, On prime fuzzy bi-ideals of semigroups, Iranian Journal of Fuzzy Systems, 7(3) (2010) 115-128.

16. F.M.Sison, Ideal theory in ternary semigroups, Math. Japon, 10 (1965) 63-84.

17. T.Vasanthi and M.Amala, Some special classes of semirings and ordered semirings, Annals of Pure and Applied Mathematics, 4(2) (2013) 145-159.

18. T.Vasanthi and N.Solochona, On the additive and multiplicative structure on semirings, Annals of Pure and Applied Mathematics, 3(1) (2013) 78-84. 